\title{
A brief on genotyping methods of hepatitis D virus
}

\begin{tabular}{|c|}
\hline $\begin{array}{l}\text { Laura Iulia Polosanu }{ }^{1} \text { Olguta Diaconu }{ }^{1} \text {, Ana Maria Buburuz², } \\
\text { Razvan Ioan Grecu }{ }^{3} \text {, Luminita Smaranda lancu }{ }^{1}\end{array}$ \\
\hline $\begin{array}{c}{ }^{1} \text { Department of Preventive Medicine and Interdisciplinarity Microbiology, Faculty of Medicine, } \\
\text { "Grigore T. Popa", University of Medicine and Pharmacy, Iasi, Romania }\end{array}$ \\
\hline $\begin{array}{l}\text { 2Department of Internal Medicine, Faculty of Medicine, } \\
\text { "Grigore T. Popa" University of Medicine and Pharmacy, Iasi, Romania }\end{array}$ \\
\hline $\begin{array}{l}\text { 3Department of Primary Care and Epidemiology, Faculty of Medicine, } \\
\text { "Grigore T. Popa" University of Medicine and Pharmacy, Iasi, Romania }\end{array}$ \\
\hline
\end{tabular}

\begin{abstract}
Hepatitis D virus (HDV) infection is known to be one of the infections that causes the most severe forms of hepatitis, having the worse outcome with higher rates of fulminant hepatitis and liver failure. At the present, there are 8 major clades of HDV known. Considering the involvement of different genotypes in the clinical outcome of hepatitis D, an ideal patient approach would contain with HDV genotyping.

The aim of this study was to better understand each HDV genotyping method described in recent years. The objectives were: to identify the techniques used for HDV genotyping, to make a comparative approach to the genotyping techniques, to appreciate the best method of HDV genotyping and to estimate the likelihood of practical application of these techniques.

In order to make a comprehensive approach on the genotyping methods of HDV, we've carried out a search on the main databases and included the studies that were original, peer-reviewed research studies and were performed on human subjects. We found 4 genotyping methods described in several studies over the years. The methods were: hybridization, RT-LAMP (Reverse Transcription-Loop-Mediated Isothermal Amplification), PCR-RFLP (Polymerase Chain Reaction- Restriction Fragment Length Polymorphism) and PCR followed by sequencing.

This study could be the basis for implementing the standard technique used in HDV genotyping.
\end{abstract}

Keywords: hepatitis D virus, genotyping, diagnosis

\section{INTRODUCTION}

\section{Target condition being diagnosed - hepatitis D}

Hepatitis D virus (HDV) infection is known to be one of the infections that causes the most severe forms of hepatitis, having the worse outcome with higher rates of fulminant hepatitis and liver failure (1). The discovery of HDV in 1977 by Rizzetto has risen many questions but not enough answers (2). At the present, there is no specific treatment against HDV infection, the Interferon-based therapy remaining the only weapon to fight against this disease $(3,4)$.
HDV is a controversial subject because of its genetic diversity that causes different clinical outcomes and influences the responses to treatment and the prognosis (5). At the present, there are 8 major clades of HDV known (numbered from 1 to 8) (6). HDV-1 is prevalent worldwide, while the rest of the clades have a specific geographical distribution: HDV-2 is more common in the Far East, Japan, Taiwan and parts of Russia; genotype 3 is predominantly found in the Amazon Basin, while genotype 4 is mainly present in Taiwan and Japan and the other genotypes 5-8 were found in African patients who migrated to Northern Europe $(7,8)$. 
HDV-1 is associated with both severe and mild forms of the disease, HDV-2 is associated with moderate clinical symptomatology, HDV-3 determines a very aggressive clinical picture and the association with genotype F of HBV (Hepatitis B Virus) has determined acute fulminant hepatitis in most cases in South America. Regarding HDV-4, there are studies showing that in some areas it has been associated with a faster progression to cirrhosis (9). Lately, many studies have suggested that the genetic diversity of HDV also influences the quantification methods of the RNA-HDV. Given that most tests use the genotype $1 \mathrm{HDV}$ as a standard in validating the methods, many of them fail to estimate the viral load in non-1-HDV genotype (10).

\section{Clinical pathway - standardization of care process}

The hepatitis D diagnosis consists of detection of total IgM anti-HDV antibodies which can be found both in acute and chronic infection. Still, several studies showed that in certain cases these types of antibodies were absent in some positive RNA-HDV samples (11). With this in mind, the safest way to diagnose HDV infection remains the quantification of RNA-HDV. In order to do this, many methods have been described over the years, some faster, others more specific, others with a lower risk of contamination (12). It's hard to decide which method is better because most are in-house techniques that use different work protocols. The main discrepancies are encountered in primers design (hepatitis D antigen or the ribozyme region) (13), in the amplification techniques (performed by manual or automatic devices, in one or two steps, using hybridization or modified TaqMan probes) $(14,15)$ and in internal standards (in vitro transcribed RNA-HDV or plasmid DNA containing HDV target sequences) $(16,17)$. Since there are so many variables, a standard RNA or cDNA quantification method is far from being implemented. This topic was the subject of many debates in recent years, and lead in 2013 to the implementation by World Health Organization (WHO) of a standard control for HDV-1 used in nucleic acid amplification technique based assays (18). However, subsequent studies shown that the assay that uses this standard control tend to underestimate the RNAHDV quantification of the other genotypes (10).
Considering the involvement of different genotypes in the clinical outcome of hepatitis D, an ideal patient approach would contain with the determination of the HDV genotype. Thereby, in this article we focus on the next step in diagnosing the HDV infection, the genotype HDV identification. As seen in viral load quantification, there is also a variation in HDV genotyping techniques. At the moment, genotyping is only used in the research approach. A rapid and efficient method of genotyping that can be used in clinical practice would improve the patient management and it could be the starting point of developing targeted therapies. Until now, there are no standard methods for HDV genotyping. Several studies shown different ways to identify the HDV clade, but we cannot say certainly which method is best.

\section{OBJECTIVES}

The aim of this study is to understand better each HDV genotyping method described in recent years. The objectives are: to identify the techniques used for HDV genotyping and to make a comparative approach in the techniques found; to appreciate the best method of HDV genotyping and to estimate the likelihood of practical application of the methods as a standard of genotyping of the RNAHDV genome.

\section{MATERIALS AND METHODS}

\section{Electronic searches}

For making a comprehensive approach about the genotyping methods of HDV, we've carried out a search of PubMed, Google Scholar, Cochrane library and Embase. Through the searching task, we entered the following "HDV genotyping", "hepatitis D genotype", "RT-PCR AND HDV", "RLFP AND HDV", "direct sequencing AND HDV", "RT-LAMP AND HDV” and „Hybridization AND HDV”.

\section{Inclusion criteria}

In order to select these methods, we have searched original, peer-reviewed studies describing genotyping techniques performed on human subjects. We didn't take into account the study protocols, reported cases, reports of experts' opinion and those that were performed on animals subjects. 


\section{RESULTS}

In our research we managed to find four genotyping methods described in several studies. The methods are: hybridization, RT-LAMP (Reverse Transcription Loop Mediated Isothermal Amplification), PCR-RFLP (Polymerase Chain Reaction Restriction Fragment Length Polymorphism) and PCR followed by sequencing (Table 1).

TABLE 1. Research studies and each method described

\begin{tabular}{|l|l|}
\hline Genotyping method & Studies \\
\hline PCR-RFLP & $\begin{array}{l}\text { Wu } 1995(19) \\
\text { Mirshafiee 2009 (20) } \\
\text { Souza 2015 (21) }\end{array}$ \\
\hline $\begin{array}{l}\text { Hybridiza on } \\
\text { microarray }\end{array}$ & Sun $2004(22)$ \\
\hline $\begin{array}{l}\text { PCR + direct } \\
\text { sequencing }\end{array}$ & $\begin{array}{l}\text { Altuğlu } 2005(23) \\
\text { Shang } 2012(24) \\
\text { Coller 2018 (25) (qRT-PCR on automated } \\
\text { pla orm) }\end{array}$ \\
\hline RT-LAMP & Wang 2012 (26) \\
\hline
\end{tabular}

\section{DISCUSSION}

Various methods have been described over the time to achieve this goal, methods from Hybridization, RT-LAMP (Restriction Fragment Length Polymorphism) to RFLP and PCR followed by sequencing. Although there are studies that analyze each method from the point of view of the accuracy and applicability in clinical laboratories, no comparison has yet been made to all these techniques.

The first method described for HDV genotyping was the hybridization technique, made by Gupta in 1989 (27). Since then, another 5 clades were identified and at least 2 genotyping techniques had been discovered (28). Genotyping by hybridization is a method that uses known sequences of DNA to hybridize a complementary sequence in the studied DNA, sequences that will be determined by fluorescent labeling. This method uses short DNA sequences (oligonucleotides) taken from a database to construct the target sequence to be identified (29).

There are no studies to identify the accuracy of the hybridization in HDV genotyping. In 1994, Gallenger et al. described a method of hybridization for quantification of HDV, and not for genotyping (30). In 2004 Sun et al. presented a combination between PCR and hybridization, resulting in a DNA microarray that offers a potential solution for diagnosis of different HDV genotypes. Hybridization is known to have a good specificity, but a low sensitivity, and PCR having an increased risk of contamination (22). As time went on, the PCRbased assays improved and now there are one-step PCR methods described having lower risk of contamination and higher sensitivity and specificity (15). In addition, Sun et al. described the method as a very simple and cost-effective technique that uses clinical diagnostic gene chips (22). Until now, there are no hybridization techniques verified on all the HDV genotypes. The advantage of this method is the ability to identify a large number of homogeneous targets.

RFLP is a simple genotyping method that cleaves the PCR products using restriction enzymes. It was first applied for HDV genotyping in 1995, when Wu et al. used Xhol and Sacll as restriction enzymes (19). Following the attachment of such enzymes, the cDNA-HDV genome is cleaved, resulting in fragments of different lengths, that once applied in the electrophoresis gel will migrate into the electric field according to their size. Thus, for each HDV genotype, a characteristic pattern of migration of the different cDNA sections of HDV will be obtained.

RFLP genotyping was compared to direct sequencing and proved to be a more effective technique for the identification of the $3 \mathrm{HDV}$ genotypes known at that time. This method proved to be helpful in the discovery of new HDV genotypes later in 1998 (31). Also, Mirshafiee et al. described this method to be a simple and reliable technique (20). Recently, in 2015, Souza et al. characterized a nested PCR-RFLP method capable to identify all HDV genotypes (21). In order to validate this technique they compared the results with the ones resulted from the phylogenetic analysis. RFLP proved to be another reliable method, easy to perform. It does not require radiolabelling and can be used for mass analysis in epidemiological and clinical studies.

PCR is the classic method of amplifying any DNA of RNA copy genome. In principle, for the amplification of the RNA genome it is recommended to convert it into the cDNA with the aid of a reverse transcriptase for greater stability of the analyzed product (18). Then, genotyping by direct sequencing involves sequencing of the entire RNAHDV genome followed by phylogenetic analysis in 
which specific sequences of each genotype are tracked, identified and stored in different banks of genes, e.g. GeneBank.

Regarding PCR followed by sequencing, several methods had been described over the years. In 2005, Altuglu et al. used a RT-PCR-sequencing method applied on chronic hepatitis infection patients from Turkey, and also described it as a reliable method (23). Then, Shang et al. proposed in 2012 a Real-Time PCR-based technique in which the amplicons of the quantitative HDV Real Time were used directly for sequencing, avoiding the steps of RNA extraction, RT-PCR and electrophoresis performed in Altuglu's study (24). Thereby, the latter seems to be a better choice in HDV genotyping considering his reduced risk of contamination. Recently, Coller et al. described an even better technique in which the extraction and amplification is being done on an automated platform.

RT-LAMP is another method of HDV genotyping described by Wang et al. in 2012 (26). Contrasted with conventional PCR and Real Time PCR, there are fewer preparation steps and less expensive equipment involved. It requires only 4 specially designed primers that can recognize the target DNA (32) and an isothermal temperature of $65^{\circ} \mathrm{C}$ for 50 minutes for the amplification reaction to take place. Therefore, the advantages of this method are simplicity, amplification efficiency and a reduced contamination risk. This method is less time consuming in contrast with PCR that needs at least 2 hours to complete the analysis. Also, it offers the possibility to identify the results by using the naked eye. In this study, Wang et al. evaluated the potential application of RT-LAMP, testing the sensitivity and specificity of the method in comparison with real-time PCR, but the exact values of the these parameters has not been calculated. The most considerable disadvantage is that this method was designed only for the identification of genotype 1 HDV (26). To sum up, this seems like a reliable method that should also be tested on the nonHDV-1 genotypes.

An accurate comparison between these methods will be hard to make considering that all these assays rely on the use of a specific in-house standard. Of all methods, hybridization and RT-LAMP could be the future for a rapid diagnosis, where there is an urgent need for results, while RFLP and PCR-sequencing seems to be the basic and reliable techniques that can be used in all laboratories for research and clinical diagnosis. RFLP remains a good choice for some undeveloped countries which do not have an available sequencing platform.

\section{CONCLUSIONS}

In conclusion, although anti-HBV infection vaccination can prevent Delta infection, in many countries chronic HDV infections represent a public health problem and earlier diagnosis is crucial. Serological markers (antigens and antibodies) are not enough for an accurate diagnosis. HDV genotyping is important because it is the only way we can better understand the clinical outcome of the patient, predict the response of the treatment and formulate a prognosis. This study could be the basis for implementing the standard technique for HDV genotyping. Further studies are needed to describe a genotyping standard of HDV and to develop techniques that could be easy to use also in practice.

\section{REFERENCES}

1. Tsatsralt-Od B., Takahashi M., Nishizawa T. et al. High prevalence of dual or triple infection of hepatitis B, C, and delta viruses among patients with chronic liver disease in Mongolia. J Med Virol 2005; 77(4):491-499.

2. Rizzetto M., Canese M.G., Arico S. et al. Immunofluorescence detection of new antigen-antibody system (delta/anti-delta) associated to hepatitis $B$ virus in liver and in serum of $\mathrm{HBsAg}$ carriers. Gut 1977;18:997-1003.

3. Alves A., Branco C., Cunhaet C. Hepatitis Delta Virus: A Pelicular Virus. Hindawi Publishing Corporation 2013;3:1-11.

4. Biçer K.Ç., Ari A., Genç V.E. et al. Efficacy of entecavir treatment among chronic hepatitis B nucleos(t)ide-naïve and -experienced patients. Turk J Med Sci 2015;45(1):99-104.

5. Deny P. Hepatitis delta virus genetic variability: from genotypes I, II, III to eight major clade. Curr Top Microbiol Immunol 2006; 307:151171.

6. Le Gal F., Gault E., Ripault M. et al. Eighth major clade for hepatitis delta virus. Emerg Infect Dis 2006;12:1447-1450.

7. Pascarella S., Negro F. Hepatitis D virus: An update. Liver Int 2011; 31:7-21.

8. Cross T.J., Rizzi P., Horner M. et al. The increasing prevalence of hepatitis delta virus (HDV) infection in South London. J Med Virol 2008; 80:277-282.

9. Heidrich B., Deterding K., Tillmann H.L. et al. Virological and clinical characteristics of delta hepatitis in central Europe. J Viral Hepat 2009;16:883-894. 
10. Su C.W., Huang C.W., Huo Y.H. et al. Genotypes and viremia of hepatitis $B$ and $D$ viruses are associated with outcomes of chronic hepatitis D patients. Gastroenterology 2006;130:1625-1635.

11. Le Gal F., Brichler S., Sahli R. et al. First international external quality assessment for hepatitis delta virus RNA quantification in plasma. Hepatology 2013; 64:1483-1494.

12. Mansour W., Malick F.Z., Sidiya A. et al. Prevalence, risk factors, and molecular epidemiology of hepatitis $B$ and hepatitis delta virus in pregnant women and in patients in Mauritania. J Med Virol 2012; 84:1186-1198.

13. Ferns R.B., Nastouli E., Garson J.A. et al. Quantitation of hepatitis delta virus using a single-step internally controlled real-time RT-qPCR and a full-length genomic RNA calibration standard. J Virol Methods 2012; 179:189-194.

14. Katsoulidou A., Manesis E., Rokka C. et al. Development and assessment of a novel real-time PCR assay for quantitation of hepatitis D virus RNA to study viral kinetics in chronic hepatitis $D$. J Viral Hepat 2013; 20:256-262 .

15. Mederacke I., Bremer B., Heidrich B. et al. Establishment of a novel quantitative hepatitis D virus (HDV) RNA assay using the Cobas TaqMan platform to study HDV RNA kinetics. J Clin Microbiol 2010; 48:2022-2029.

16. Kodani M., Martin A., Mixson-Hayden T. et al. One-step real-time PCR assay for detection and quantitation of hepatitis D virus RNA. J Virol Methods 2013; 193:531-535.

17. Scholtes C., Icard V., Amiri M. et al. Standardized one-step real-time reverse transcription-PCR assay for universal detection and quantification of hepatitis delta virus from clinical samples in the presence of a heterologous internal-control RNA. J Clin Microbiology 2012; 50:2126-2128.

18. Gal F., Gordien E., Affolabi D. et al. Quantification of hepatitis delta virus RNA in serum by consensus real-time PCR indicates different patterns of virological response to interferon therapy in chronically infected patients. J Clin Microbiol 2005; 43:2363-2369.

19. Chudy M., Hanschmann K.M., Bozday M. et al. Collaborative Study to Establish a World Health Organization International Standard for Hepatitis D Virus RNA for Nucleic Acid Amplification Technique (NAT)-Based Assays. WHO Press 2013;WHO/BS/2013.2227.

20. Gupta S., Valinluck B., Govindarajan S. Detection of Hepatitis Delta Virus in Serum and Liver Tissue by Molecular Hybridization Validation of a Rapid Spot-Hybridization Technique. Am J Clin Pathol 1989; 92(2):218-221.

21. Hughes S.A., Wedemeyer H., Harrison P.M. Hepatitis delta virus. Lancet 2011; 378:73-85
22. Preparata F.P., Upfal E. Sequencing-by-hybridization at the information-theory bound: An optimal algorithm. J Comput Biol 2000; 7(3):621-630.

23. Gallagher M., Morris T.T., Fields H.A. Chemiluminescent hybridization assay for hepatitis delta virus RNA in serum. $J$ Clin Microbiol 1994; 32(4):1112-1114.

24. Sun Z.H., Zheng W.L., Zhang B. et al. Detection of hepatitis D virus by cDNA microarray method. Hepatobiliary Pancreat Dis 2004; 3(3):423-427.

25. Wu J.C., Choo K.B., Chen C.M. et al. Genotyping of hepatitis D virus by restriction-fragment length polymorphism and relation to outcome of hepatitis D. Lancet 1995; 346:939-941.

26. Wu J.C., Chiang T.Y., Sheen I.J. Characterization and phylogenetic analysis of a novel hepatitis $D$ virus strain discovered by restriction fragment length polymorphism. J Gen Virol 1998; 79:1105-1113.

27. Mirshafiee H., Mahmoodian-Shooshtari M., Sharifi Z. et al. Genotype analysis of hepatitis delta virus from hepatitis B surface antigen-positive patients using PCR-RFLP in Tehran. Arch Iran Med 2009; 12(3):238-243.

28. Botelho-Souza L.F., Souza V.D., Oliveira Dos Santos A. et al. Characterization of the Genotypic Profile of Hepatitis Delta Virus: Isolation of HDV Genotype-1 in the Western Amazon Region of Brazil. Intervirology 2015; 58(3):166-171.

29. Altuğlu I., Ozacar T., Sertoz R.Y. et al. Hepatitis delta virus (HDV) genotypes in patients with chronic hepatitis: molecular epidemiology of HDV in Turkey. Int J Infect Dis 2007;11:58-62.

30. Shang D., Hughes S.A., Horner M. et al. Development and validation of an efficient in-house real-time reverse transcription polymerase chain reaction assay for the quantitative detection of serum hepatitis delta virus RNA in a diverse south London population. J Virol Methods 2012;184:55-62.

31. Wang C., Shen X., Lu J. et al. Development of a reverse transcription-loop-mediated isothermal amplification (RT-LAMP) system for rapid detection of HDV genotype 1. Lett Appl Microbiol 2013; 56(3):229-235.

32. Notomi T., Okayama H., Masubuchi H. et al. Loop-mediated isothermal amplification of DNA. NAR 2000; 28(12):e63.

33. Coller K.E., Butler E.K., Luk K.-C. et al. Development and performance of prototype serologic and molecular tests for hepatitis delta infection. Scientific Reports 2018; 8:2095.

34. Ferns R.B., Nastouli E., Garson J.A. Quantitation of hepatitis delta virus using a single-step internally controlled real-time RT-qPCR and a full-length genomic RNA calibration standard. J Virol Methods 2012; 179:189-194. 